\title{
Exploring gaps in surveillance of a small measles outbreak in Toronto, Canada
}

\author{
Gournis $E^{1,2^{*}}$, Shane $A^{1,3}$, Shane $E^{1}$, Arthur $A^{1}$, Berger $L^{1,2}$
}

\begin{abstract}
In early 2015, an outbreak of 10 confirmed measles cases occurred in Toronto, Ontario. As part of the outbreak response, the Toronto Public Health staff conducted both traditional and supplementary case investigation activities. Despite this extensive effort, and unlike many previous measles outbreaks in Canada, neither the source case nor any confirmed epidemiologic links between cases were identified. The outbreak investigation brought to light potential gaps in the current measles surveillance and suggested approaches to future investigations: routine use of social media and other time-stamped resources to enhance case investigation; early and repeated targeted communication with primary care partners to improve case detection; and continued efforts to increase and maintain sufficient immunization coverage to interrupt transmission.
\end{abstract}

\author{
Affiliations \\ ${ }^{1}$ Communicable Disease Control, \\ Toronto Public Health, Toronto, \\ ON \\ 2 Dalla Lana School of Public \\ Health, University of Toronto, \\ Toronto, ON \\ ${ }^{3}$ Canadian Field Epidemiology \\ Program, Public Health Agency of \\ Canada, Ottawa, ON
}

^Correspondence: egourni@ toronto.ca

Suggested citation: Gournis E, Shane A, Shane E, Arthur A, Berger L. Exploring gaps in surveillance of a small measles outbreak in Toronto, Canada. Can Comm Dis Rep 2016;42:146-8. https://doi.org/10.14745/ ccdr.v42i07a02

\section{Introduction}

On January 28, 2015, Toronto Public Health was notified of a laboratory-confirmed measles case. Reports of nine additional confirmed cases soon followed. Molecular evidence supported the hypothesis that all the cases resulted from a single importation, but this could not be confirmed through epidemiologic evidence.

This article explores the gaps in measles detection and investigation identified while investigating this outbreak, describes the supplementary epidemiologic activities used to address these gaps, and considers the implications for future outbreak response activities.

\section{The outbreak}

A detailed description of the epidemiology and public health response to this outbreak will be provided elsewhere (S. Thomas et al. Measles outbreak with unique genotyping). To summarize, 10 confirmed and genotypically similar measles cases were reported to Toronto Public Health between January 28, 2015, and February 17, 2015. An incident management system was activated to manage and coordinate the outbreak response activities. All cases and contacts were investigated and managed as per Ontario's Infectious Diseases Protocol (1). This included follow-up of 1,548 contacts and hosting of 10 post-exposure prophylaxis clinics. No secondary cases were detected among contacts. Routine case investigation information did not reveal the source of the measles outbreak.

\section{Supplementary epidemiologic investigations}

As it became apparent that epidemiologic links between the cases were missing, Toronto Public Health epidemiologists conducted supplementary activities during the public health investigation. The purpose of these activities was to increase the completeness of the information about possible exposure provided by the cases in order to better understand and characterize measles transmission within the community. First, a subset of cases or their guardians were asked to use any social media information (e.g., Instagram posts) and online banking records from their respective accounts to help recall activities during the potential acquisition and transmission periods. Each supplementary phone interview took an additional two to three hours per case (including the time required to validate addresses). This led to a number of additional locations of interest beyond those ascertained in the initial interviews. In addition, the investigation used the social network visualization tool Pajek (2) to identify overlapping exposures. As the exposure list expanded and manual review became time-consuming and onerous, including social network visualization in the routine reporting cycle led to a quicker and more systematic method to identify potential epidemiologic links.

\section{Gaps in detection}

Despite using traditional and supplementary case investigation measures, there remained insufficient evidence to confirm the source of the outbreak or any epidemiologic links between cases. Given that the National Microbiology Laboratory characterized the cases as genotypically similar and likely due to a single importation event (Personal communication, Alberto Severini, National Microbiology Laboratory, March 31, 2016), a gap in case detection and investigation was evident. While missing the source case of an outbreak is 
not uncommon in Canada, a small and contained community outbreak of measles with no identified epidemiologic links between cases is unusual (3).

Several hypotheses could explain these findings. It is possible that people with measles never presented to the health care system. Alternatively, they may have presented but were not identified as suspect measles cases and not tested appropriately. In both these scenarios people may have experienced attenuated, or subclinical, symptoms, possibly as a result of secondary vaccine failure (4), and did not meet the classical clinical or laboratory case definition for measles. It is also possible that the traditional and supplementary epidemiologic case investigation processes and tools used in this investigation were insufficient to identify the common exposure(s) given the high transmissibility of the virus, the mobility of the population and the urban environment in which the cases resided. The social media-facilitated reinterviews were only conducted using a subset of cases. Had these methods been applied to all 10 cases additional links may have been found.

\section{Discussion}

Despite routine and enhanced investigations of an outbreak of measles limited to 10 laboratory-confirmed cases in Toronto, Ontario, neither the source nor any confirmed epidemiologic links were identified. It is important to consider the implications of these findings for measles surveillance and Canada's elimination efforts.

In order to sufficiently document Canada's measles elimination status, it is essential to minimize the number of sporadic or unknown source cases and ensure thorough investigation and understanding of transmission events. While it has been suggested that subclinical cases may be less infectious than clinical cases, detection of all cases remains important in order to understand outbreak and transmission dynamics (5).

Although Canada experiences few and small outbreaks of measles, the resources required to manage these outbreaks are considerable, specifically in terms of follow-up of persons under investigation, suspect cases and contact management in the context of high vaccine coverage (6). For example, after the outbreak was declared, Toronto Public Health received many reports of persons under investigation that did not meet the outbreak case definition yet required substantial public health and laboratory resources to rule out. Finding a balance between an acceptable level of surveillance sensitivity required to characterize and interrupt transmission and the appropriate allocation of resources required to maintain that level of sensitivity is a key challenge, especially in areas with high and homogeneous immunization coverage.

The gaps identified in this summary suggest there are important opportunities to improve case identification and epidemiologic investigation of measles. The resources for any additional activities need to be weighed against what is already required to meet the current measles outbreak investigation standards.

To improve the ability to accurately describe the epidemiology of measles among confirmed cases, continued emphasis on the collection of comprehensive exposure (both acquisition and transmission) information during case interviews is needed. This could include routinely asking cases to review time-stamped resources (e.g., bank and credit card statements or social media sites) to help remind them of their activities and locations during their exposure and communicability periods and using social networking visualization to deal with the complexity of this added information.

To address the potential gap in sensitivity identified through this outbreak investigation, early, repeated and active communication with primary care and emergency department networks during measles outbreaks may help reinforce the key signs and symptoms that trigger appropriate laboratory testing procedures. This was done during the 2015 outbreak via alerts and communications with primary care providers. If fewer reports of persons under investigation are received because physicians know whom to report and test, public health resources could be reallocated to additional epidemiologic activities. To improve detection of measles cases during non-outbreak periods (i.e., in order to detect source cases), it is important to remind health care providers that symptoms may be attenuated in previously immunized people and to consider travel history from measles-endemic areas to inform the differential diagnosis.

Continued efforts to increase immunization coverage and access to electronic records confirming immunization status may allow public health to both rely on herd immunity to interrupt measles transmission following an importation and shift the balance of public health resources from contact management to persons under investigation as well as suspect and confirmed cases.

\section{Conclusion}

Despite routine and supplementary case investigation activities, Toronto Public Health could not confirm any epidemiologic links between the 10 outbreak cases. The gaps in case detection and investigation revealed by this unusual outbreak can inform future outbreak response activities.

\section{Acknowledgements}

We would like to acknowledge all the staff at Toronto Public Health who contributed to the investigation and management of this outbreak, the team at Public Health Ontario who helped coordinate the provincial investigation and response, and the staff at the National Microbiology Laboratory for their work and information on the phylogenetic characterization of the cases.

\section{Conflict of interest}

None.

\section{Funding}

None. 


\section{References}

1. Ministry of Health and Long-Term Care. Infectious diseases protocol. Appendix A: disease-specific chapters. Chapter: Measles. Toronto (ON): The Ministry [revised 2014 Aug]. http://www.health.gov.on.ca/en/pro/programs/publichealth/ oph_standards/docs/measles_chapter.pdf.

2. Batagelj $\mathrm{V}$, Mrvar A. Pajek - program for large network analysis. 2000. http://vlado.fmf.uni-lj.si/pub/networks/pajek/.

3. Public Health Agency of Canada. Elimination of measles, rubella and congenital rubella syndrome in Canada: documentation and verification report. Ottawa (ON): Public Health Agency of Canada [Last modified April 4, 2013]. http://www.phac-aspc.gc.ca/im/vpd-mev/measles-rougeolemrer-eng.php.

4. De Serres G, Boulianne N, Defay F, Brousseau N, Benoît $M$, Lacoursière $S$, et al. Higher risk of measles when the first dose of a 2-dose schedule of measles vaccine is given at 12-14 months versus 15 months of age. Clin Infect Dis 2012;55(3):394-402.
5. Glass K, Grenfell BT. Waning immunity and subclinical measles infections in England. Vaccine 2004;22(29-30):41106.

6. Wilson SE, Fediurek J, Seo CY, Deeks SL, Lim GH. Immunization coverage report for school pupils: 2012-13 school year. Toronto (ON): Public Health Ontario; 2014. https://www.publichealthontario.ca/en/eRepository/ Immunization_coverage_report_2012-13.pdf.

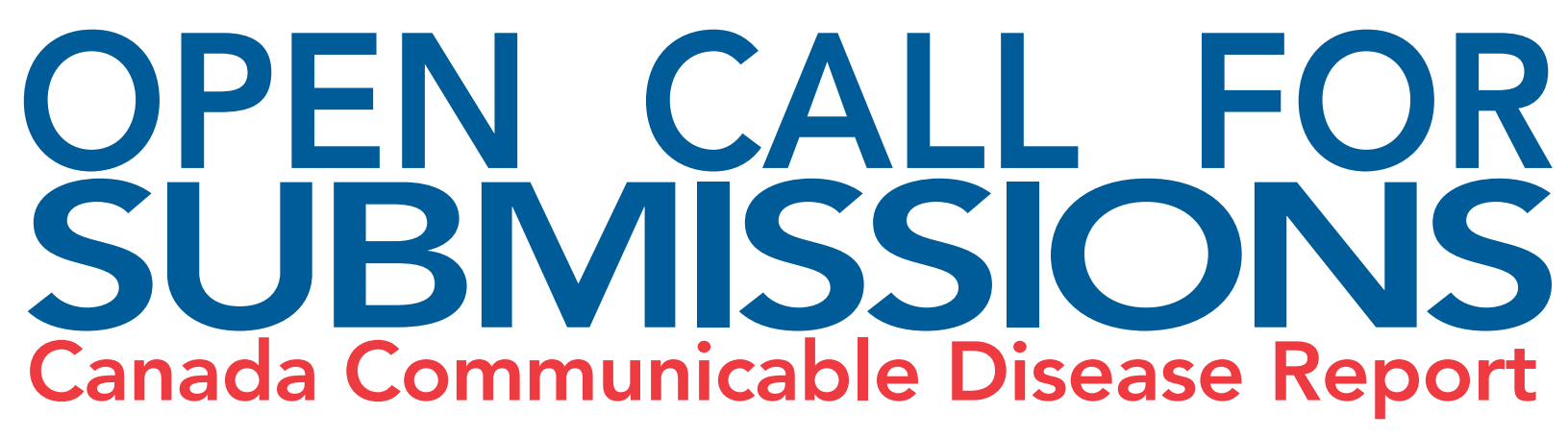

In 2015, we published articles with authors from almost all provinces and territories.

We welcome submissions of manuscripts with practical, authoritative information on infectious diseases that will inform policy, program and practice.

\section{Visit: www.phac-aspc.gc.ca/publicat/ccdr-rmtc/ia-ra-eng.php}

Public Health Agence de la santé

Agency of Canada publique du Canada

Canadă 